\title{
A conceptual model of public medical service system based-on cell phone mobile platform
}

\author{
Hongjiao $\mathrm{Fu}^{1}$, Yue Zhao \\ 1 Department of Economic Information Management, School of Information, \\ Renmin University of China, Beijing 100872, P.R. China \\ \{zhao.yue, fuhj\}@ruc.edu.cn
}

\begin{abstract}
In recent years, cell phones have played an increasingly important role in rapidly-developing global telecommunication services. At present, mobile business develops very fast. However, the development in other mobile service fields, such as public service, mobile medical service, etc, is still in its infant stage. Drawing on the experience of the 'doctor workstation project' which is cooperated by Renmin University of China and Norway Fredskorps Corporation, this paper discusses the research and implementation of the Doctor Workstation System based on cell phone mobile platform. From the practice of the Doctor Workstation System, the paper advances a conceptual model of public medical service system based-on cell phone mobile platform.
\end{abstract}

\section{Research Background}

Along with the revolution of global information technology and the vigorous development of mobile communication technology, cell phones have played an increasingly important role in rapidly-developing global telecommunication services. Consequently, mobile services are fast becoming an integral part of people's life. At present, mobile business, which is one of typical representative form of mobile services, develops very fast. Pertinent data show that in recent years development of mobile business will produce a leap in quality. It is estimated that the value brought by global mobile business will exceed 0.2 billion US dollars in 2007[1].

However, the development in other mobile service fields, such as public service, mobile medical service, mobile government affairs, etc, is still in its infant stage. Their application scope and depth remain very limited. The knowledge and acceptance of these services by the public are extremely low.

Drawing on the experience of the 'doctor workstation project' which is cooperated by Renmin University of China and Norway Fredskorps Corporation, this paper discusses the research and implementation of the Doctor Workstation System

Please use the following format when citing this chapter:

I'U, H., Thao, Y., 2007, in IIIP Intemational lederation for Information Processing, Volume 252, Intcgration and Imnovation Orient to E-Society Volume 2, eds. Wang, W., (Boston: Springer), pp. 88-95. 
based on cell phone mobile platform. From the practice of the Doctor Workstation System, the paper advances a conceptual model of public medical service system based-on cell phone mobile platform.

\section{Introduction to doctor workstation system based-on cell phone mobile platform}

\subsection{Introduction to doctor workstation project}

Two operation parties of this project are our University and Norges Fredskorps Corporation. Our goal is to establish an integrated doctor workstation system which consists of two parts: traditional web browser and doctor workstation system basedon cell phone mobile platform which is the key point of the paper.

Now whether in developing or developed countries, doctor workstation system based-on information technology all has different degree application. However, among them, most systems are based-on traditional browser. Although this kind of systems have high technology maturity and users are very familiar with them, this way of implementation which is unitary and influenced largely by equipment limitation already cannot adapt demands of every aspects in today when mobile services, mobile application develop very fast and mobile demand are very high. Under this situation, we put forward doctor workstation system based-on cell phone mobile platform which will effectively make full use of flexibility and convenience of cell phone mobile platform to overcome shackles of traditional doctor workstation system and provide medical service to doctors at all times and places[1,2].

\subsection{Doctor workstation system based-on cell phone mobile platform}

\subsubsection{The function of system}

The prominent characteristics of doctor workstation system based-on cell phone mobile platform are flexibility and convenience, however, the limitations of cell phones, such as processing capacity of CPU, storage capacity, space of main memory and interactive ability, restrict implementation of system function to a great extent. In short, with regard to doctor workstation system based-on cell phone mobile platform, we cannot expect and do not need to pursue integrity of system function. We will realize function modules which are suitable for cell phone mobile platform through which doctors can complete their work with high efficiency.

After logging in the system by cell phone, the doctor can do the following works:

\section{(1) Case inquiring}

Through connecting distant database, users will inquire related case data within their authorities, and then the system will transfer the inquired result back to cell phone platform to the doctors. So doctors will not limited by the space and time, they can inquire cases and know the situation of their patients at any time and places. They also can revise or delete case information and input information of new cases, which extremely increases efficiency and saves human resources. The procedures of case inquiring are shown in Fig. 1. 


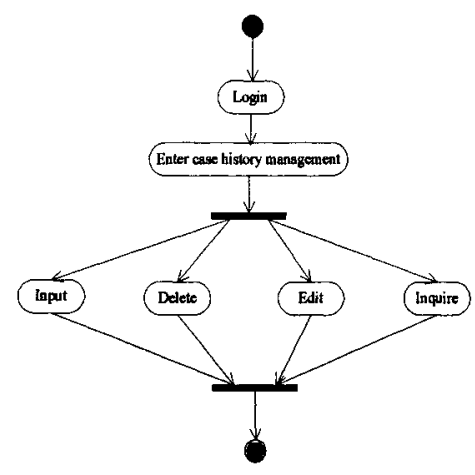

Fig. 1. The procedure of case inquiring

\section{(2) Consultation management}

Consultation management is the most distinguishing featured function module in the system, which realizes the function of inter-consultation about cases and patient's conditions between doctors. The system adopted sending-receiving mechanism which is similar to a simple email system to realize this function. Consultation between the doctors is created, distributed and processed similarly to the way of email.

\section{- Creating consultation}

The doctor logs in the system, enters consultation management interface and creates a new consultation. After the doctor finishes filling in consultation information, he can lead in cases information and medical images related to this consultation as attachments, for example, related X-Ray image, etc. The process of creating a new consultation is shown in Fig. 2.

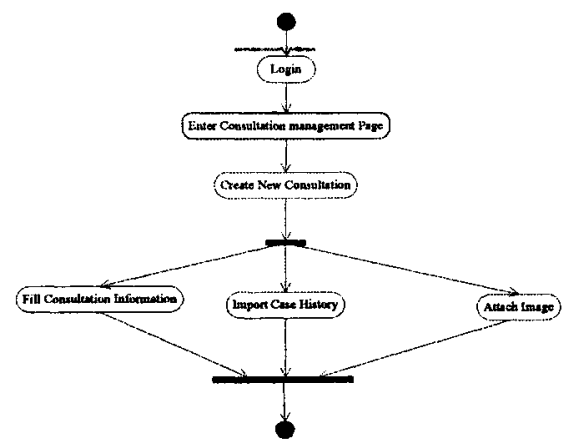

Fig. 2. The process of creating a new consultation 


\section{- Distributing consultation}

After creating a new consultation, distributing consultation can be made. There are two types of distributing consultation: (1) point-to-point. On the premise of having a clear target, the system will transfer the consultation in the way of point-topoint, which means directly sending the consultation to the receiver who asked for it. For example, a difficult and complicated case of illness can be sent to an expert in this field to ask for advice. (2) mass-distributing. In some cases, the user does not have a clear consultation target, but need a mass line and listening to more useful opinions from the experts. In this case, we can use the way of mass-distributing. We can select a series of experts and send them the consultation to obtain more opinions.

In the way of mass-distributing, the system also supports a special way called "public consultation", i.e. we don't select persons specially designated for a post when creating a consultation, but mark this consultation as a public consultation in this field. In this way, all users in this field can browse current public consultations by checking public consultation column and choose questions they interested.

\section{- Responding consultation}

After receiving consultation information from other users, the user can respond by the system. As to the consultation in the way of point-to-point, obviously the way of response is aimed at single user. As to the consultation in the way of massdistributing, two ways of response can be selected. One is to send the response directly to the sender, which is similar to the way of point-to-point. Another one is to send the response oriented towards the public, in which all users who concern this consultation topic can see related condition of discussion, and which will take effect of knowledge exchanging and sharing.

\section{(3) Image processing}

In the information of cases, it is more probable to contain image information, for example, X-ray photographs, Nuclear Magnetic Resonance (NMR) photographs, etc, so the system should have basic functions of image processing.

The basic functions of image processing realized by the system include: function of zooming whole or partial image, function of marking image comparison, and function of trimming, etc.

(4) Multi-language support

In order to facilitate system's international application and dissemination in the future, the system supports multi-language operation. Users can select one language when they $\log$ in.

\subsubsection{The architecture of system}

In general, this system is composed of two parts, a J2ME-based portal and a J2EE-based application server [2-4]. The architecture of system is shown in Fig. 3.

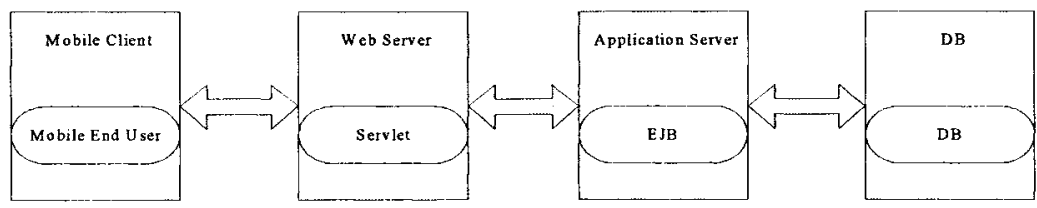

Fig. 3. The architecture of system 
The web server is responsible for handling the request of the end user. The web server receives the request from the end user and sends it to the application server. The EJB container is responsible for interacting with database and it handles most of the application logic. We make full use of the benefits of the EJB container, for example, the support for the transaction management, the data persistency and the guarantee for high security level.

\subsubsection{The communication mechanism of system}

(1) The way of communication

Considering the CPU capacity and the support way of communication of mobile devices, we choose the widely used lightweight HTTP protocol as the way of communication.

(2) Data format

In order to release the network transfer pressure, save the storage space and reduce the transfer delay, we choose the binary as the format of the data [2].

(3) The security of communication

Because the J2EE application server and the J2ME platform both support the SHTTP based on the SSL, we can transfer the data in encrypt to communicate with server.

\subsubsection{The features of system}

Firstly, the most important advantage of the system is flexibility [3, 5]. Doctor workstation system based-on cell phone mobile platform could be accessed by the doctor ubiquitously, which breaks the limitation of space and time. This is one of the crucial factors for the service quality of the doctor workstation system and this is also the core concept of the mobile services which embodies the value of the system.

Secondly, the support of multi-language which is also one of the important items for the system usability makes the system easy for international application and dissemination in the future.

\section{A conceptual model of public medical service system based-on cell phone mobile platform}

By taking an example of corporation project of our University and Norway Fredskorps Corporation, the paper discussed doctor workstation system based-on cell phone mobile platform and its functions and the system architecture.

Today, with the high speed development of the mobile application and mobile service, every walk of life will consider whether this industry could serve in mobile way as one of the important factors of the service quality, which is more important in medical service industry.

Currently, the information technology has widely used in the medical service industry and its application has already come to a certain level, for example, the application of the management information system in the hospital. We believe that with the high speed development of the mobile application and service, it is certain that the mobile application will widely be used in the medical service industry $[6,7]$.

Now we will discuss the conceptual model of public medical service system based-on cell phone mobile platform specifically. 


\subsection{The characteristic of the system}

\subsubsection{Agility}

Agility is the essential and distinguishing characteristic of the system and the reason why a mobile application exists [8].

(1)The easy acquirement of the application and the compatibility of the client software

The end user could get the application software easily by the way of web download, mobile device based GPRS download and so on.

The client software should have strong compatibility and not rely on special mobile devices and operating systems. The mainly used operating systems in the mobile device now are Symbian, Windows CE and Linux which three are now sharing the market. So the client software should have these three versions at least.

(2) Usability of the system

This is mainly concerned with the human-computer interaction, for example, the Graphical User Interface (GUI), the response delay time and so on. We will not discuss further on this.

\subsubsection{The open interface}

The prevalence of the mobile application is an inevitable trend and it will be widely used in every face of life. This requires us be well prepared for the cosmically forthcoming application in the future. In order to establish an integrated mobile service system, the system should have open interface for the future connection with other systems, for example, the data interface with the bank, human resources management department and supervision departments, etc.

\subsubsection{Security}

It is obvious that the system security is very important, because there are lots of personal privacy and secrecy data. Beside the security measures we usually take, we must pay more attention to the danger of data filched when the data is transferred between the database and mobile device.

In the security area, we should guarantee the following facts: (1) the resources of the system can't be accessed without authorization; (2) the user can't access the resources of the system that are not included in his authorization; (3) make sure that the resource of the system can't be filched during the data transformation; (4) well prepared for the disaster backup.

We are going to take the measures that integrate the information technology and the management rules. The information technologies used include the identity validate, the software fire-wall, and the data encryption; the management rules include the proper assigning of the access authorization, the data restore and backup, and auditing the system periodically.

\subsubsection{Internationalization}

The system should have the function of internationalization and could easily switch to different versions between different languages and custom. Moreover, we should use the international standard for the specialty term, which makes it easy for the future international communication, for example, we should use the International Classification of Diseases version 10 (ICD10) as the only identity of the diseases. 


\subsection{Conceptual model of the system of public medical service system based-on cell phone mobile platform}

The conceptual model of the system of public medical service system based-on cell phone mobile platform we put forward is shown in Fig. 4. We will describe every module of the model as follow [8-10]: (1) The patients communicate with the doctor by the system to inquire about their illness and update the doctor's advice. (2) The patients finish the payment through the interface with the bank. (3) The patients make appointment with the doctor through the medicine treatment interface. (4) The patients purchase the medicine from the medicine vendor through the medicine seller interface. (5) The doctors make consultations with each other by the mobile device.

(6) The doctor can inquire the records of cases and update them by the mobile device. (7) The functional department could supervise the behaviors of the related parts to avoid illegal operation and could also inspect the plague and make warnings and take measures in time.

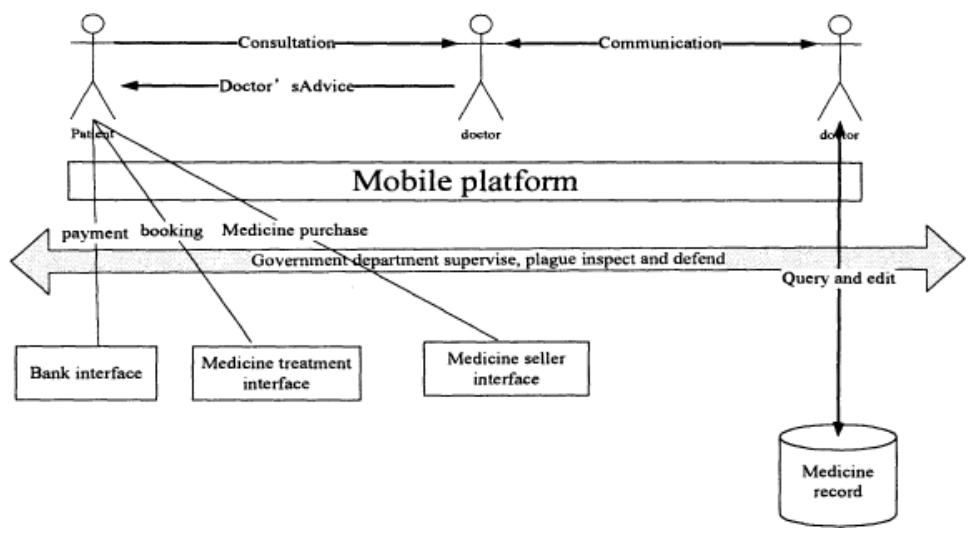

Fig. 4. The conceptual model of the system of public medical service system based-on cell phone mobile platform

The working mechanism of the model indicates the following two features: (1) The strong interactive communication ability: The model not only integrates the old systems but builds an agile platform for the communication of the users including the doctors and patients. They could make the communications easily through the system. We even could integrate the instant messaging system into our platform to maximize the communion feature of the system. This is not only about the cost and the efficiency but concerns the quality of the health and the life. (2) Coordinate management: This model integrates the bank, medical treatment and the medicine seller system which could be managed coordinately. This feature avoids the emergence of the information gaps and the information isolated. Mean while, the government department could handle the supervision responsibility simply through this model which saves lots of human resource and time. 


\section{Summary}

This paper discusses the system architecture, functions and benefits of the doctor workstation based-on cell phone mobile platform. And then we put forward a conceptual model of the system of public medical service system based-on cell phone mobile platform, and illustrate its characteristic.

We believe that, with the fast development of the mobile application, public medical service system based-on cell phone mobile platform will gradually come into practice in the near future.

\section{Reference}

1. C.X. Fan and J.W. Zou, "Research and design for a mobile application integrated delivery platform", Journal of Beijing University of Posts and Telecommunications ,27, 202-206 (2004).

2. P. Tarasewich, "Designing mobile commerce applications", Communications of the $A C M, 46(12), 57-60(2003)$.

3. C. Ryan and A. Gonsalves, "The effect of context and application type on mobile usability: an empirical study", Proceedings of the Twenty-eighth Australasian conference on Computer Science, 115-124(2005).

4. D. Narayanan, J. Flinn, and M. Satyanarayanan, Using history to improve mobile application adaptation, Third IEEE Workshop on Mobile Computing Systems and Applications, 61-66(2000).

5. P. Abrahamsson, A.Hanhineva, H. Hulkko, T. Ihme, J. Jaalinoja, M. Korkala, J. Koskela, P. Kyllonen and O. Salo, "Mobile-D: an agile approach for mobile application development", Companion to the 19th annual ACM SIGPLAN conference on Object-oriented programming systems, languages, and applications, 174-175(2004).

6. T. Zrimec, "A content-based retrieval system for medical images", Proceedings of 20027 th International Conference on Control, Automation, Robotics and Vision, 180-185(2002).

7. Y.F. Chen, H. Huang, J.R. John, S. Jora, S.Reibman and A.B. Wei, "Personalized multimedia services using a mobile service platform", Proceedings of 2002 IEEE Wireless Communications and Networking Conference 918- 925(2002).

8. G.A. Bolvary and S. Kis, "Computer based information/advisory/alert system for the Hungarian healthcare professionals", Biomedical Engineering Days, Proceedings of the 1992 International, 134-136(1992).

9. N. Houssos, A. Alonistioti, L. Merakos, M. Dillinger and M. Fahrmair, "Advanced adaptability and profile management framework for the support of flexible mobile service provision", Wireless Communications, IEEE [see also IEEE Personal Communications],52-61(2003).

10. A. Pfitzmann, B. Pfitzmann and M. Schunter, "Trusting mobile user devices and security modules", Computer,30(2), 61-68(1997). 